and Dr. Jules Carret has just published an ingenious book on the subject. ${ }^{1}$

The paper, of which the following is an abstract, is an attempt to investigate the results of the supposition that the earth is slowly changing its shape from internal causes. The first part is devoted to the mathematical consideration of the precession and nutations of a spheroid slowly undergoing such a change. It is shown that the obliquity of the ecliptic must have remained sensibly constant throughout geological history, and that even gigantic polar icecaps cannot have altered the position of the Arctic circle by so much as three inches; and this would be the most favourable redistribution of matter for producing that effect.

But a slow distortion of the earth would displace the principal axis of figure of the earth, and the axis of rotation would always sensibly follow the axis"of figure. Thus the result would be a change in the geographical position of the poles, without any alteration of the diameters of the arctic circles, or in the width of the tropics.

For reasons, which cannot be given here, it is maintained that the earth would not be rigid enough to resist the effects of considerable departures from the figure of equilibrium, such as would arise from a wandering of the pole of figure from its initial position ; and that readjustments to an approximate form of equilibrium would probably take place, at considerable intervals of time, impulsively by means of earthquakes. Such periodical adjustments would not sensibly modify the geographical path of the principal axis as due to terrestrial deformation.

The rest of the paper is given to the consideration of the kinematical question of the change in the geographical position of the pole, due to any distortion of the earth. It is assumed, in the first place, that the deformation is such that there is no change in the strata of equal density; and accordingly all suppositions as to the nature of the internal changes accompanying geological upheaval and subsidence are set aside. The forms of continents and depressions are investigated, which would cause the maximum deflection of the pole for elevations and depressions of given amounts.

In order to make numerical application to the case of the earth, some estimate is required of the extent to which it may have become distorted during any one geological period. From the consideration of certain facts, the author believes that from $\frac{1}{10}$ to $\frac{1}{2} \delta$ of the whole earth's surface may have, from time to time, undergone a consentaneous rise or fall ; and that the vertical rise or fall may be about 10,000 feet, or rather equivalent to about I0,000 feet, when allowance is made for the influx of the sea into depressed areas.

The first application given in this paper is to continents and seas of the most favourable shapes and positions. It may be here stated that if $\frac{1}{00}$ of the earth's surface is elevated by 10,000 feet, the deflection of the pole is II $1^{\prime} ;$ if $\frac{1}{20}$ of the whole surface, $\mathrm{I}^{\circ} 46 \frac{1}{2}^{\prime}$; if $7^{\frac{\pi}{0}}$, $3^{\circ} 17^{\prime}$; and if $\frac{1}{2}, 8^{\circ} 4 \frac{1^{\prime}}{2},{ }^{2}$ In each case an equal area is supposed to fall simultaneously.

Other examples are also given for continents and seas which do not satisfy the maximum condition; in some the boundaries are abrupt cliffs, in others shelving.

The conclusion is arrived at that a single large geological change, such as those which obtain on the earth, is competent to produce an alteration in the position of the pole of from one to three degrees of latitude, on the hypothesis that there is no change in the law of internal density.

Various other hypotheses as to the nature of the internal changes accompanying the deformation of the earth are discussed.

I. It is shown that if upheaval and subsidence are due

I "Le Déplacement Polaire." Savy, Paris, 1877 . America about "033 of
2 The area of Africa is about "059, and of South Amera the earth's surface. to a shrinking of the earth as a whole, but to the shrinking being quicker than the mean in some regions and slower in others, the results are the same as those previously attained.

2. The increase of surface-matter due to the deposit of marine strata also gives the same results.

3. The hypothesis that upheaval and subsidence are due to intumescence or contraction immediately under the regions in question is considered. Under certain special assumptions, too long to recapitulate, it is shown that the previous results must be largely reduced. It appears that if the swelling or contracting stratum is tolerably thin and at all near the surface, the deflection of the pole is reduced to quite an insignificant amount. Even if the intumescence extends right down to the centre of the earth in a cone bounded by the elevated region, the results would be only about $\frac{2}{3}$ of the former ones. Hence it appears that the earlier results can only be stated as the greatest possible for given superficial changes.

In conclusion it is pointed out that if the earth be quite rigid, no redistribution of matter in new continents could ever cause the deviation of the pole from its primitive position to exceed the limit of about $3^{\circ}$. But if the previously maintained view is correct, that the earth readjusts itself periodically to a new form of equilibrium, then there is a possibility of a cumulative effect; and the pole may have wandered some $10^{\circ}$ or $15^{\circ}$ from its primitive position, or have made a smaller excursion and returned to near its old place. No such cumulation is possible, however, with respect to the obliquity of the ecliptic.

It is suggested that possibly the glacial period may not have been really one of great cold, but that Europe and North America may have been then in a much higher latitude, and that on the pole retreating they were brought back again to the warmth. There seem to be, however, certain geological objections to this view.

\section{THE NEW STAR IN CYGNUS ${ }^{1}$}

$\mathrm{N}$ January 9 the sky was unusually clear and the spectrum of Dr. Schmidt's Nova came out with amazing sharpness and brilliancy. In addition to the five bright lines seen on the 2 nd, two others were detected, viz. :-

No. Ia W. L. 594 Very narrow line.

, $7 \quad 4$ I4 E Excessively faint, but still certainly and repeatedly seen.

Between wave-lengths 655 and 594 the spectrum was certainly banded, and, most probably, there were two additional faint maxima of brilliancy in that interval. The continuous spectrum attains a maximum in the region about W. L. 525 , and extends, though possibly not without interruption, as far as the faint line No. 7. The star was estimated of seventh magnitude, and was of a red colour with a decided tinge of purple, reminding me forcibly of the varieties of red produced by the quartzplate in Zöllner's photometer. RALPH COPELAND

Lord Lindsay's Observatory, Dunecht, January I3

\section{OUR ASTRONOMICAL COLUMN}

THE New COMET.-.-The comet discovered by M. Borrelly, at Marseilles, on the morning of February 9, appears to have been iound independently by Herr Pechille at the Observatory of Copenhagen on the following morning. During the past week it has been making a pretty near approach to the earth, and had the weather been more favourable in Europe, it would probably have been very generally observed.

The following elements of the orbit have been calculated by Mr. Hind from the first observation by M. Borrelly, one at 
Kiel on the Ioth, and one made at the Imperial IObservatory at Strasburg on the $15 \mathrm{th}$, and communicated by Prof. Winnecke:Perihelion Passage, January 19.1801\%, Greenwich M.T.

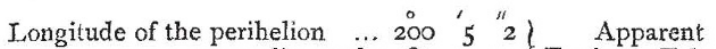
In ", ascending node $\left.187 \begin{array}{rrr}5 & 22\end{array}\right\}$ Equinox, Feb. Io. Inclination to the ecliptic $\quad \ldots \quad 27 \quad 5 \quad 13$

Logarithm of perihelion distance 9.907086

Heliocentric motion-retrograde.

According to this orbit the comet was distant from the earth, at the time of discovery, $0^{\circ} 45$, the earth's mean distance from the sun being taken as unity. Its apparent path in the heavens about the perigee, and up to March 6 , will be sufficiently defined by the following ephemeris : -

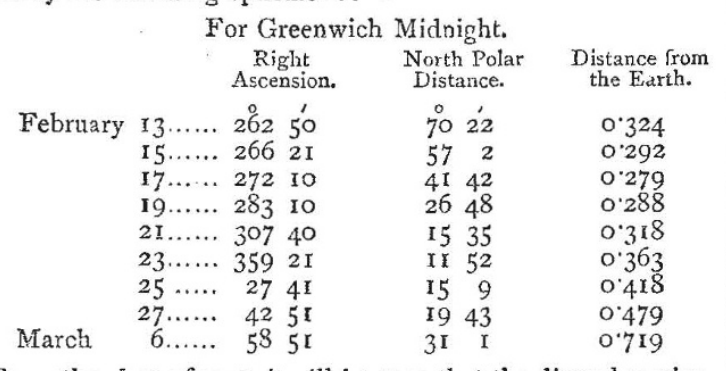

From the above figures it will be seen that the diurnal motion, about the middle of the present month, amounted to $8^{\circ}$ in arc of great circle ; the comet was nearest to the earth soon after midnight on the $\mathrm{I} 7 \mathrm{th}$. At the time of feribelion passage it would be situated about $6^{\circ}$ to the east of Antares, distant from the earth $x \cdot 15$.

There is a certain similarity, but by no means a striking one, between the elements of this comet and those of the comet of I590, observed by Tycho Brahé, the orbit of which was first calculated by Halley, and, in 1846 , after a new reduction of Tycho's observations, by Mr. Hind (Ast. Nach., No. 584). It may be worthy of remark that shortly after the passage of the ascending node, the comet of 1590 approaches very near to the orbit of the planet Venus, the least distance not exceeding $0^{\circ} 04$. Still the differences between the elements of the comets of 1590 and 1877 , especially in the perihelior distance, are material.

The "Berliner Astronomisches Jahrbuch."--Under the active superintendence of Prof. Tietjen, the Berliner Fahrbuch continues the heavy labour involved in the preparation of ephemerides of the extensive group of small planets, a work which for many years past has heen made its specialty. In the volume for 1879 now before us, we have the places for 1877 of 151 out of the 172 actually known members of the group, No. 164, Eva, being the latest discovery included in the list. Also the elements of their orbits and the opposition magnitudes, a very useful addition towards the identification of these minute bodies amongst the fainter stars. The portion of the volume devoted to the small planets extends to 106 pages. The judicious transfer of the ephemeris of the moon, derived from Hansen's Tables from our Nautical Almanac, which appears between one and two years earlier, to the pages of the Berliner Fahrbuch, after adaptation to the meridian of Berlin, whereby a most serious expenditure of time and labour is saved, has already been noted in this column; it is a step which no doubt assists materially in completing the peculiar work of the fahrbuch.

The following are names which have been recently proposed, for discoveries made within the last few years: No. r $39 \mathcal{F}_{u \in w a}$ 149 Medusa, I50 Nuwa, I55 Scylla, I60 Una, 161 Athor, 163 Erigone, 164 Eva. No. 162 is not yet named.

Only four of these planets (in addition to two of the old members) attain $8.5 \mathrm{~m}$. or upwards at their oppositions in 1877 . Ariadne, in opposition on July 24, approaches the earth within about 0.84 of the earth's mean distance from the sun, and Iris, which at her opposition on November 18 , is calculated to be $6.8 \mathrm{~m}$. or on the limit of acute unaided vision, will be distant from us 0.86 -affording an opportunity which may be utilised for attempting direct measures of her diameter, though if we are not mistaken some pretty satisfactory 'measures were made at a favourable opposition a few years since, with a powerfal refractor in this country. This planet when near the earth has a decidedly red light; at two oppositions within the last fifteen years it might be identified amongst the neighbouring stars by this cir. cumstance alone.

\section{CHEMICAL NOTES}

ABSORPTION OF LIGHT IN THE BLOOD.--In a number of cases of unintentional poisoning caused by carbon monoxide in Berlin during the past winter, oxygen has been used as an antidote. Dr. Baeblich, of Berlin, lately showed the desirability of the method by means of spectroscopic proof in a public lecture. As is well known, the spectrum of blood shows two well-defined bands between Fraunhofer's lines D and $\mathrm{E}$. By the absorption of CO the position of these bands is very slightly changed in the direction of the red part of the spectrum. The difference is more strikingly shown by the addition of sulphide of ammonium. In the case of healthy blood the two bands of the spectrum disappear and are replaced by a single one situated midway between the positions of the former pair. Blood poisoned with $\mathrm{CO}$ shows no change in the bands by the same treatment. If oxygen is, however, added to it before the reduction with sulphide of ammonium, the characteristic spectrum of healthy blood is at once produced.

Physical Properties of Gallium.-M. Lecoq de Buis baudran has introduced a new method for the extraction of this metal, and has investigated some of its physical properties. Its crystalline form is octahedral ; the mean of six experinients gave as its melting point $30 \times 5$. Its specific gravity is $5^{\circ} 95^{6}$; when fused it has a silver lustre, but on solidifying it shows a tinge of blue, losing its brilliancy. It is hardly acted on by nitric acid when diluted with an equal bulk of water.

Potassium Tri-rodide. - Mr. G. S. Johnson has recently published an investigation on this body, which is prepared when a strong solution of potassium iodide is saturated with iodine, and the resulting liquid allowed to evaporate slowly over oil of yitriol. The crystals are sometimes long and isolated, sometimes appearing as hexagonal plates exhibiting a stepped arrangement like those of potassium iodide. They have a steel-blue lustre, are very deliquescent, fusing at about $45^{\circ} \mathrm{C}$., and have a specific gravity of 3.498 . When the temperature is raised above $100^{\circ}$, iodine is freely evolved from the crystals, a white mass of potas. sium iodide alone remaining. On analysis the crystals yielded $90^{\circ} 2$ to $90^{\circ} 4$ Fer cent. of iodine and 9.2 per cent. of potassium; the theoretical quantities required, supposing the body to be $\mathrm{KI}_{3}$, are iodine 90.692 per cent. and potassium 9.307 per cent. An excess of water decomposes potassium tri-iodide, with precipitation of the most of the iodine ; the crystals, however, may be dissolved in small quantities of water or alcohol, and recrystallised over sulphuric acid.

Solution of Gases in Iron, Steel, and Manganese.MM. Troost and Hautefeuille have published in the Ann. Chim. Phys., [5] vii., a reprint of their researches on this subject, previously published in other journals. When cast iron is fused in contact with silica or silicates, carbonic oxide is produced by the action of the iron carbide on silica; the iron thus becomes richer in silicon, the carbon diminishing. Melted cast iron seems to occlude considerable quantities of hydrogen, this occlusion being increased by the presence of manganese and climinished by the presence of silicon. Carbonic oxide is not taken up to so great 